\title{
Association of two synonymous splicing-associated CpG single nucleotide polymorphisms in calpain 10 and solute carrier family 2 member 2 with type 2 diabetes
}

\author{
MARIA KARAMBATAKI ${ }^{1,2}$, ANDIGONI MALOUSI ${ }^{1}$, GEORGIOS TZIMAGIORGIS ${ }^{1}$, \\ CONSTANTINOS HAITOGLOU $^{1}$, AIKATERINI FRAGOU ${ }^{1}$, ELISAVET GEORGIOU ${ }^{1}$, \\ FOTEINI PAPADOPOULOU ${ }^{2}$, GERASIMOS E. KRASSAS ${ }^{2}$ and SOFIA KOUIDOU ${ }^{1}$ \\ ${ }^{1}$ Laboratory of Biological Chemistry, Department of Medicine, Aristotle University of Thessaloniki, Thessaloniki 54124;
${ }^{2}$ Department of Endocrinology, Diabetes and Metabolism, Panagia General Hospital, Thessaloniki 55132, Greece
}

Received August 31, 2016; Accepted November 11, 2016

DOI: $10.3892 /$ br.2016.833

\begin{abstract}
Coding synonymous single nucleotide polymorphisms (SNPs) have attracted little attention until recently. However, such SNPs located in epigenetic, CpG sites modifying exonic splicing enhancers (ESEs) can be informative with regards to the recently verified association of intragenic methylation and splicing. The present study describes the association of type 2 diabetes (T2D) with the exonic, synonymous, epigenetic SNPs, rs3749166 in calpain 10 (CAPN10) glucose transporter (GLUT4) translocator and rs5404 in solute carrier family 2, member 2 (SLC2A2), also termed GLUT2, which, according to prior bioinformatic analysis, strongly modify the splicing potential of glucose transport-associated genes. Previous association studies reveal that only rs5404 exhibits a strong negative T2D association, while data on the CAPN10 polymorphism are contradictory. In the present study DNA from blood samples of 99 Greek non-diabetic control subjects and 71 T2D patients was analyzed. In addition, relevant publicly available cases (40) resulting from examination of 110 Personal Genome Project data files were analyzed. The frequency of the rs3749166 A allele, was similar in the patients and non-diabetic control subjects. However, AG heterozygotes were more frequent among patients (73.24\% for Greek patients and $54.55 \%$ for corresponding non-diabetic control subjects; $\mathrm{P}=0.0262$; total cases, 52.99 and $75.00 \%$, respectively; $\mathrm{P}=0.0039)$. The rs5404 $\mathrm{T}$ allele was only observed in CT heterozygotes (Greek non-diabetic control subjects, $39.39 \%$ and Greek patients, 22.54\%; $\mathrm{P}=0.0205$; total cases,
\end{abstract}

Correspondence to: Professor Sofia Kouidou, Laboratory of Biological Chemistry, Department of Medicine, Aristotle University of Thessaloniki, Aristotle University Campus, Thessaloniki 54124, Greece

E-mail: kouidou@auth.gr

Key words: synonymous $\mathrm{CpG}$ polymorphisms, bioinformatics analysis, heterozygosity, family history, HbA1c, CAPN10, SLC2A2
34.69 and $21.28 \%$, respectively; $\mathrm{P}=0.0258)$. Notably, only one genotype, heterozygous AG/CC, was T2D-associated (Greek non-diabetic control subjects, $29.29 \%$ and Greek patients, $56.33 \%$; $\mathrm{P}=0.004$; total cases, 32.84 and $56.58 \%$, respectively; $\mathrm{P}=0.0008)$. Furthermore, $\mathrm{AG} / \mathrm{CC}$ was strongly associated with very high $(\geq 8.5 \%)$ glycosylated plasma hemoglobin levels among patients $(\mathrm{P}=0.0002$ for all cases). These results reveal the complex heterozygotic SNP association with T2D, and indicate possible synergies of these epigenetic, splicing-regulatory, synonymous SNPs, which modify the splicing potential of two alternative glucose transport-associated genes.

\section{Introduction}

RNA splicing is a fundamental process, which contributes to the structural and functional complexity of proteins and influences their regulatory role and tissue specificity $(1,2)$. Splicing enhancers in exons are considered to be responsible for the inclusion of exonic sequences in the gene transcript. There is growing evidence that polymorphisms in high impact exonic splicing enhancers (ESEs) strongly influence the activity of disease-associated genes and modify their association with different pathological conditions (3). Bioinformatic resources are available for evaluating the efficiency of ESEs (4).

It has been previously demonstrated that a major role of intragenic DNA methylation is associated with the regulation of alternative splicing $(5,6)$. It should therefore be expected that polymorphisms, which modify a $\mathrm{G}$ or a $\mathrm{C}$ in a $\mathrm{CpG}$ dinucleotide, affect the epigenetic profile in exonic sequences that are most frequently found to be methylated (7). In sites of tentative DNA methylation, particularly when located in ESEs, this would lead to allele-specific methylation differences (8). In view of the recently demonstrated impact of DNA methylation on the splicing process (6), it is also expected that exonic $\mathrm{CpG}$ polymorphisms may further affect splicing. The presence of ESEs and their relative potential are predictable by bioinformatic analysis. Recent experimental evidence verified the consistency of the computational results with experimentally observed exon inclusion using a minigene (9). It is also evident that CpG-single nucleotide polymorphisms (SNPs) in 
prominent ESEs of disease-associated genes are of particular importance $(10,11)$. Based on this evidence, various studies have focused on genetic variations (SNPs) at CpGs, which may be responsible for predisposition to various pathological conditions, including type 2 diabetes (T2D) $(11,12)$.

T2D is a metabolic disorder characterized by high glucose blood levels associated with insulin resistance and relatively low levels of insulin. Together with obesity, blood hypertension and hyperlipidemia, T2D is one of the most frequent conditions associated with metabolic syndrome, which is currently considered a major cause for cardiovascular disease. Genetic association studies for the identification of SNPs associated with these diseases are performed by genome-wide association study (13). However, the distinct epigenetic/splicing-associated role of these SNPs has not, to the best of our knowledge, been addressed, despite previous evidence that the expression of different splicing isoforms is a major factor for disease association even in the heterozygous state $(14,15)$.

In view of the above, a bioinformatic analysis of synonymous SNPs in all T2D-associated genes (11) was performed in the present study to identify prominent CpG-SNPs, which introduce major modifications in the splicing potential of exonic sequences, which may be responsible for T2D. This analysis identified two principle CpG-SNPs, rs5404 in solute carrier family 2, member 2 (SLC2A2), and rs3749166 in calpain (CAPN10), a membrane protease, which is involved in glucose transporter (GLUT)4 translocation (16). rs3749166 (A>G) is located in exon 11 of the CAPN10 gene and rs5404 $(\mathrm{C}>\mathrm{T})$ in exon 5 of the SLC2A2 gene. The two CpG-SNPs introduce pronounced changes in the ESE score (splicing potential) of the corresponding exonic sequences in these genes. The association of CAPN10 SNP with T2D in particular, has been addressed in previous studies (17-22).

In the present study, the association of these two epigenetic CpG-SNPs were analyzed, which introduced the greatest changes of the splicing potential in the corresponding genes, with T2D and other metabolic syndrome-associated pathological conditions (arterial hypertension and obesity). In addition, the possibility that this association might be observed only in the heterozygotic state of these SNPs was investigated.

\section{Materials and methods}

Study population. The investigated population included 99 non-diabetic control participants (Table IA) and 71 T2D patients (Table IB). Participants were classified as having T2D based on the American Diabetic Association criteria (23) as follows: i) $\geq 126 \mathrm{mg} / \mathrm{dl}$ fasting plasma glucose concentration; ii) glycosylated plasma hemoglobin $(\mathrm{HbAlc}) \geq 6.5 \%$; iii) insulin use; iv) use of other diabetes medication. All participants provided their medical family history, smoking habits and dietary information, followed by written informed consent. Their names were anonymized prior to study completion. The methods followed in the present study were performed according to the Declaration of Helsinki.

The present study was approved by the Bioethics Committee of Aristotle University Medical School (Thessaloniki, Greece; protocol no. 2629; 19 April 2011), the Scientific Council of Thessaloniki Panagia General Hospital (Thessaloniki, Greece; protocol no. A9825; 9 June 2011) and the Research
Committee of Aristotle University, Operational Program 'Education and Lifelong Learning' of the National Strategic Reference Framework (NSRF) - Research Funding Program: Heracleitus II (project no. 87113).

Anthropometric and biochemical analysis. Anthropometric measurements, including weight and height were obtained according to standardized protocols. The epidemiological profile consisted of age, gender, metabolic family history, smoking status, dietary conditions, and accompanying diseases (arterial hypertension and hyperlipidemia). Participants were classified as having an accompanying disease (arterial hypertension and hyperlipidemia) when the use of antihypertensive or antihyperlipidemic medication was reported respectively, independently of their biochemical lipid profile determination. Information regarding the type of medication (tablets and insulin) and potential diabetic complications were recorded for the diabetic patients.

The biochemical analysis included determination of fasting plasma glucose, HbA1c, total serum cholesterol, low-density lipoprotein (LDL) cholesterol, high-density lipoprotein (HDL) cholesterol and serum triglycerides. Peripheral blood samples (2 ml) from all 170 participants for molecular genetic analysis were collected in tubes containing EDTA and centrifuged at $4,500 \mathrm{x} g$ for $20 \mathrm{~min}$ at room temperature. Buffy coat leukocytes were then isolated and stored at $-20^{\circ} \mathrm{C}$.

DNA extraction and genotype analysis. Genomic DNA was extracted from the buffy coat fraction prepared as described above using PureLink Genomic DNA kit (Invitrogen; Thermo Fisher Scientific, Inc., Waltham, MA, USA), according to the manufacturer's instructions. DNA integrity was verified by gel electrophoresis $(70 \mathrm{~V} / \mathrm{cm}$ for $20 \mathrm{~min})$ using $0.8 \%$ agarose gel and ethidium bromide staining. DNA purity was determined by the optical density $(\mathrm{OD})_{260} / \mathrm{OD}_{280} \mathrm{~nm}$ absorption ratio using an Eppendorf Biophotometer. Genomic sequences containing SNPs (rs3749166 and rs5404) were amplified by DNA polymerase chain reaction (PCR) using Platinum Taq DNA polymerase (Invitrogen; Thermo Fisher Scientific, Inc.). The PCR conditions for rs3749166 amplification were as follows: $94^{\circ} \mathrm{C}$ for $2 \mathrm{~min}, 35$ cycles of $94^{\circ} \mathrm{C}$ for $45 \mathrm{sec}, 60^{\circ} \mathrm{C}$ for $45 \mathrm{sec}$ and $72^{\circ} \mathrm{C}$ for $1.5 \mathrm{~min}$ followed by $72^{\circ} \mathrm{C}$ for $10 \mathrm{~min}$. A forward primer (5'-CAGGTCCCAGAGGGTGGAA-3') and a reverse primer (5'-CAGGTAGGTGGAGGGCACAA-3') were used for amplifying a 153-bp fragment containing SNP rs3749166. A 344-bp fragment containing SNP rs5404, was amplified by PCR using a forward primer (5'-TCAGGGAGGGGCTTTCATTC-3') and a reverse primer (5'-CAGTCAGGGAGGGACGAGA-3') under the following conditions: $94^{\circ} \mathrm{C}$ for $2 \mathrm{~min}, 35$ cycles of $94^{\circ} \mathrm{C}$ for $45 \mathrm{sec}, 58^{\circ} \mathrm{C}$ for $45 \mathrm{sec}$ and $72^{\circ} \mathrm{C}$ for $1.5 \mathrm{~min}$ followed by $72^{\circ} \mathrm{C}$ for $10 \mathrm{~min}$. Primer design was facilitated by Primer-BLAST (https://www.ncbi.nlm.nih.gov/tools/primer-blast/), an online primer designing tool (24). Twelve microliters of each PCR product were separated $(70 \mathrm{~V} / \mathrm{cm}$ for $20 \mathrm{~min})$ on a $2 \%$ agarose gel and visualized using ethidium bromide staining. In addition, the PCR products were purified using a PureLink PCR Purification kit (Invitrogen; Thermo Fisher Scientific, Inc.), according to the manufacturer's instructions. The sequence of the purified PCR products was verified by commercial sequence analysis (VBC-Biotech Service GmbH, Vienna, 


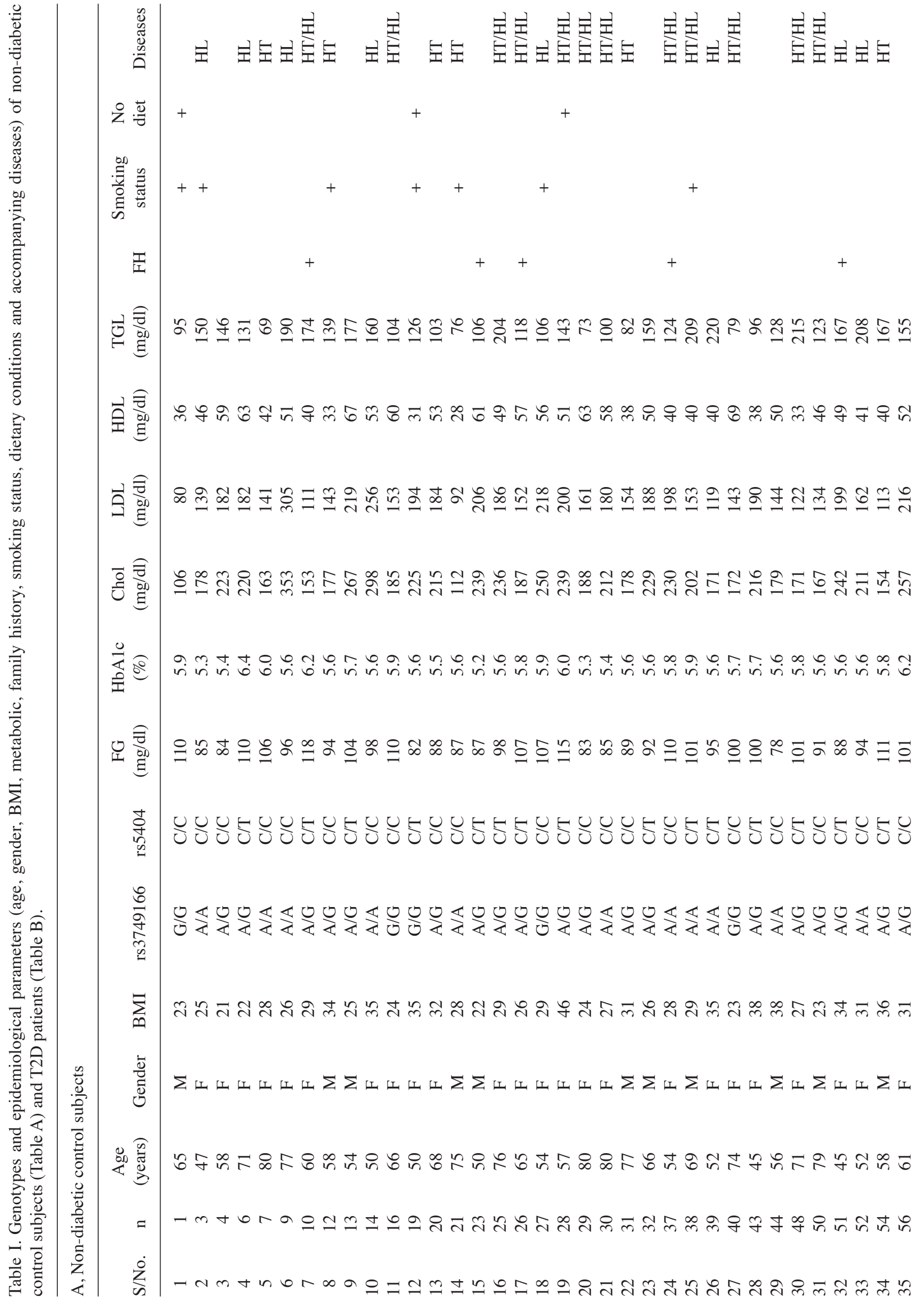




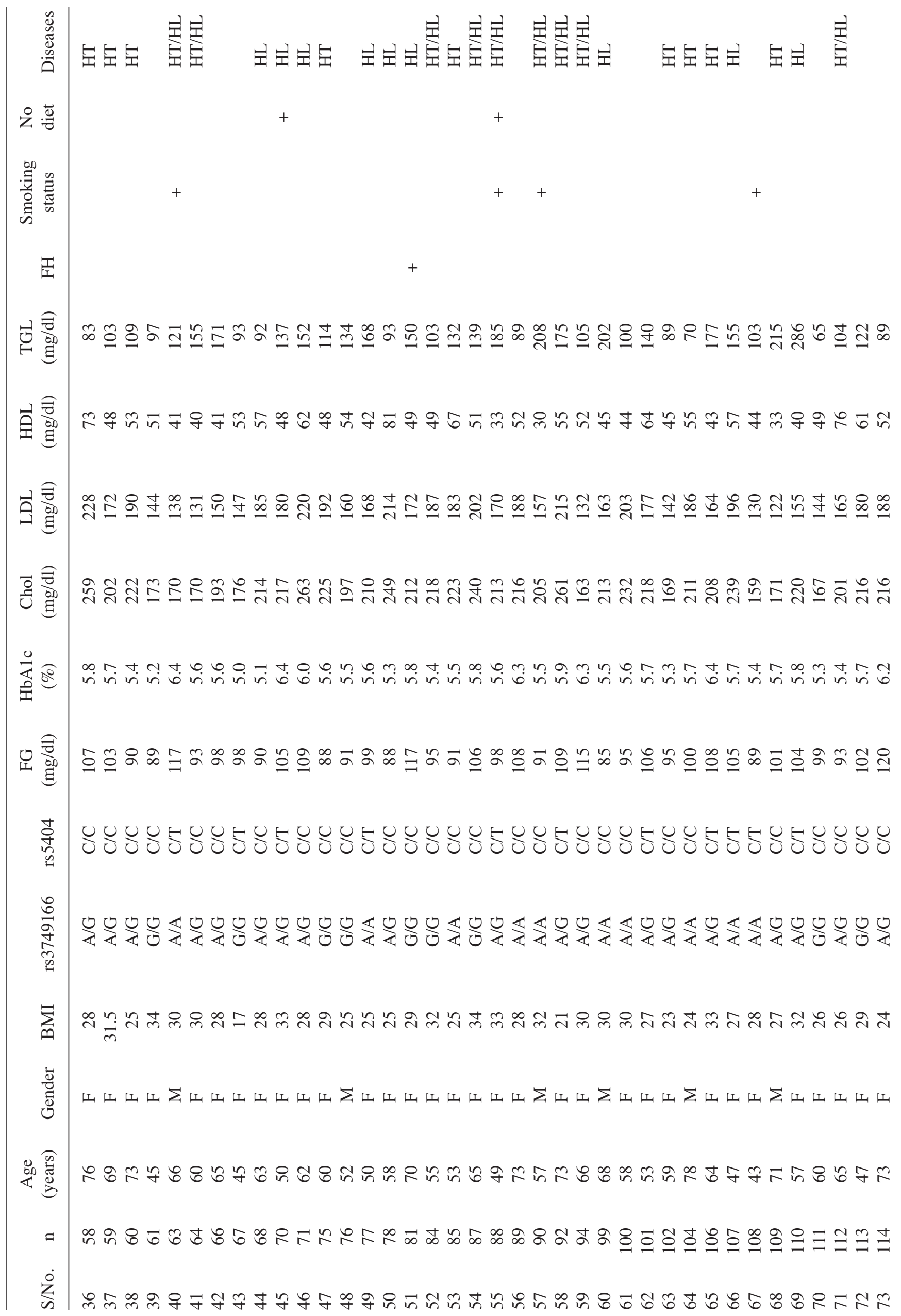




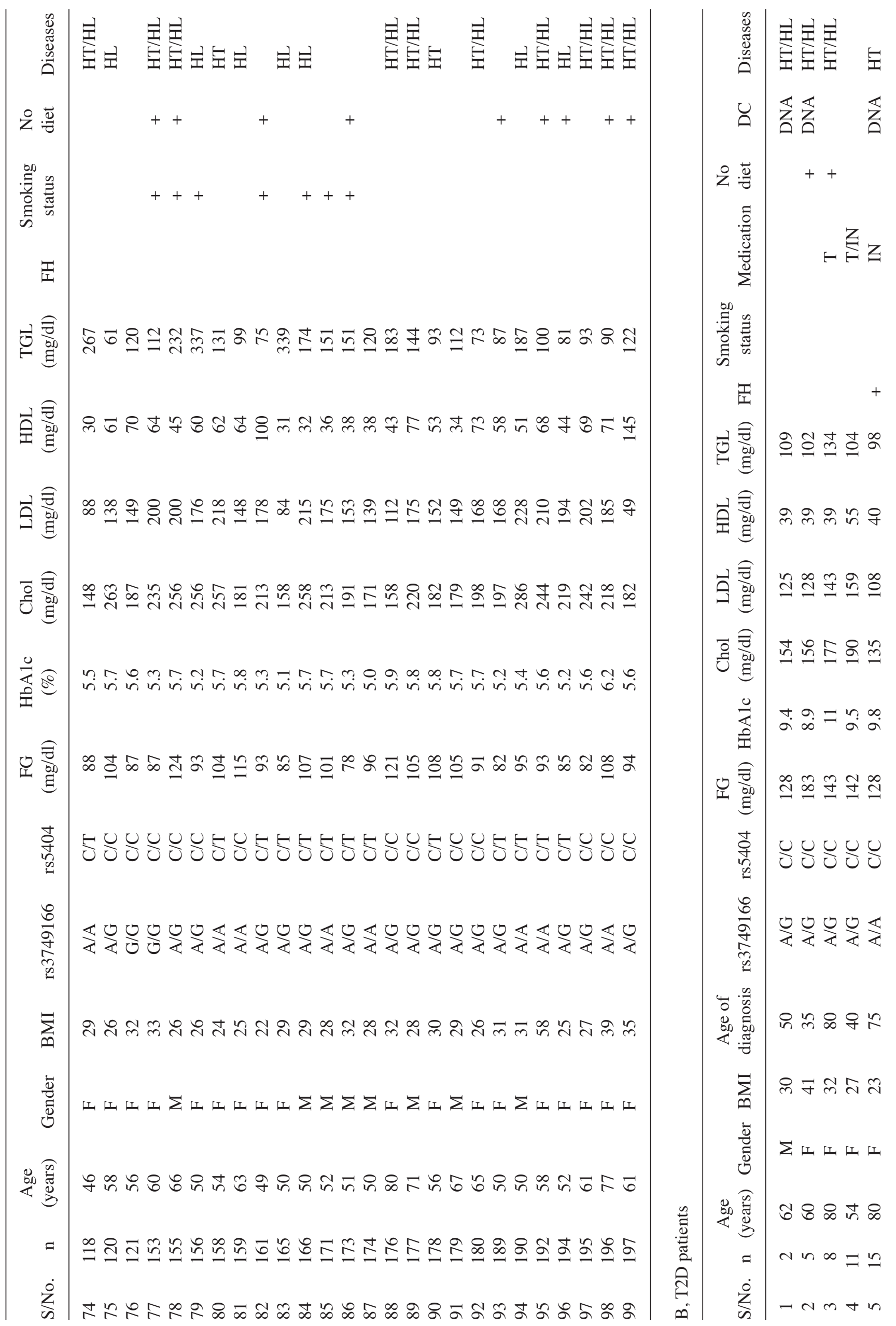




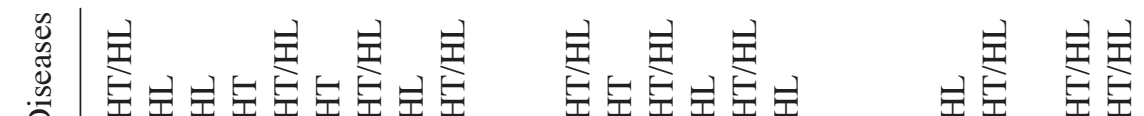

Q

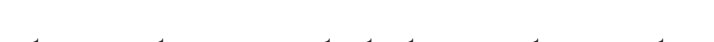

บ

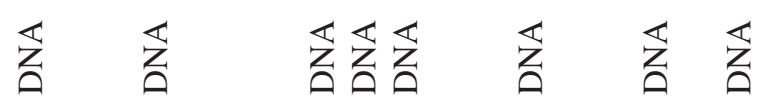

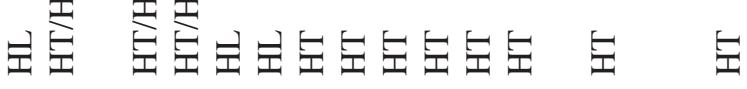

z

章

$+$

$++$

范䓃

范范茫

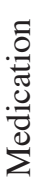

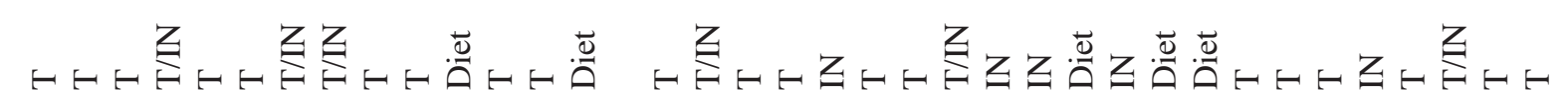

衰

TI

읍

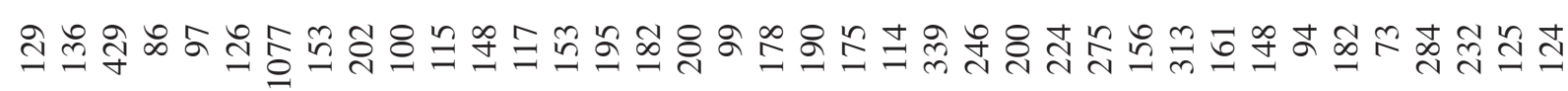

$\overrightarrow{\hat{\theta}} \frac{\hat{\theta}}{00}$

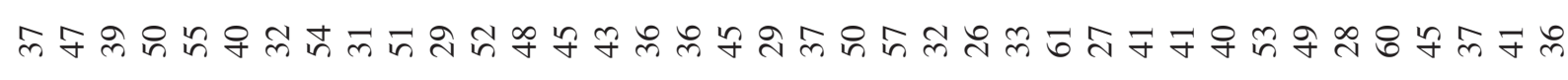

今.

三央古

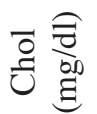

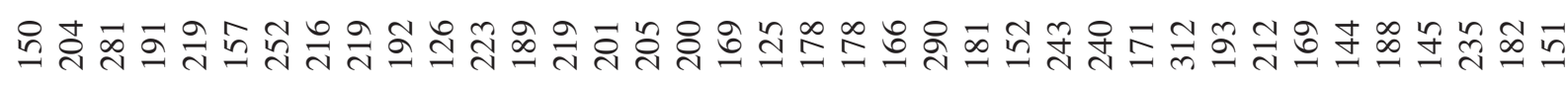

0

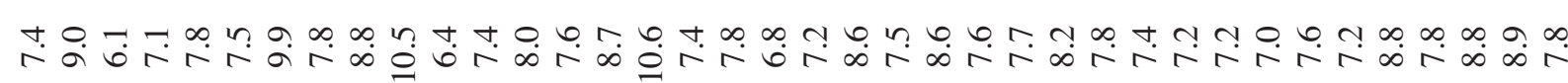

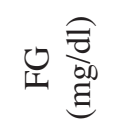

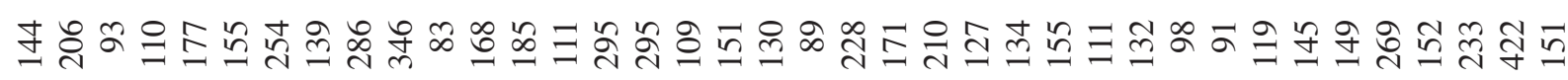

索

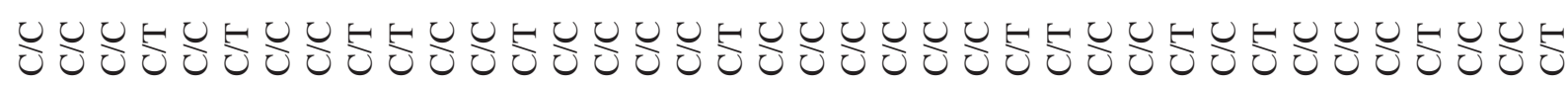

$\underset{8}{8}$

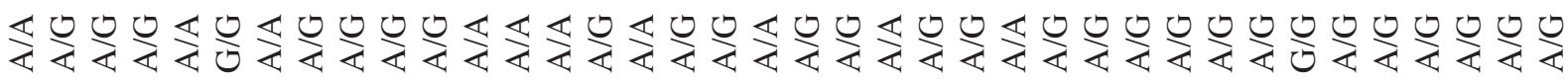

$+\frac{\pi}{0} \cdot \frac{n}{0}$

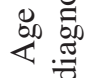

ำำำ

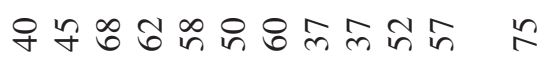

$\sum_{\infty}^{F}$

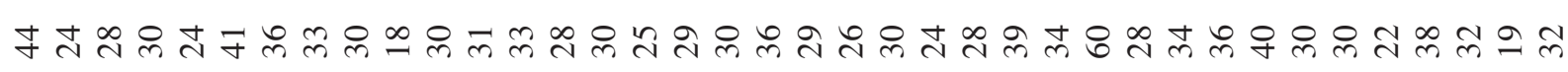

$\frac{\dot{\bar{v}}}{\overline{0}}$

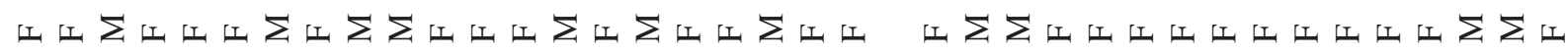

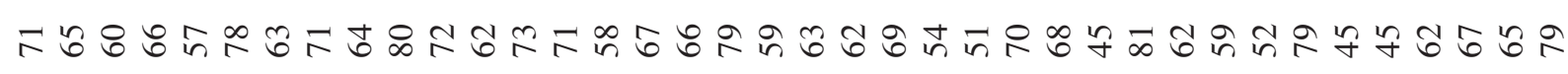

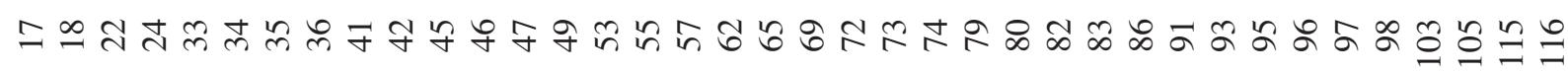

$\sum_{i=1}^{\dot{z}}$

or 


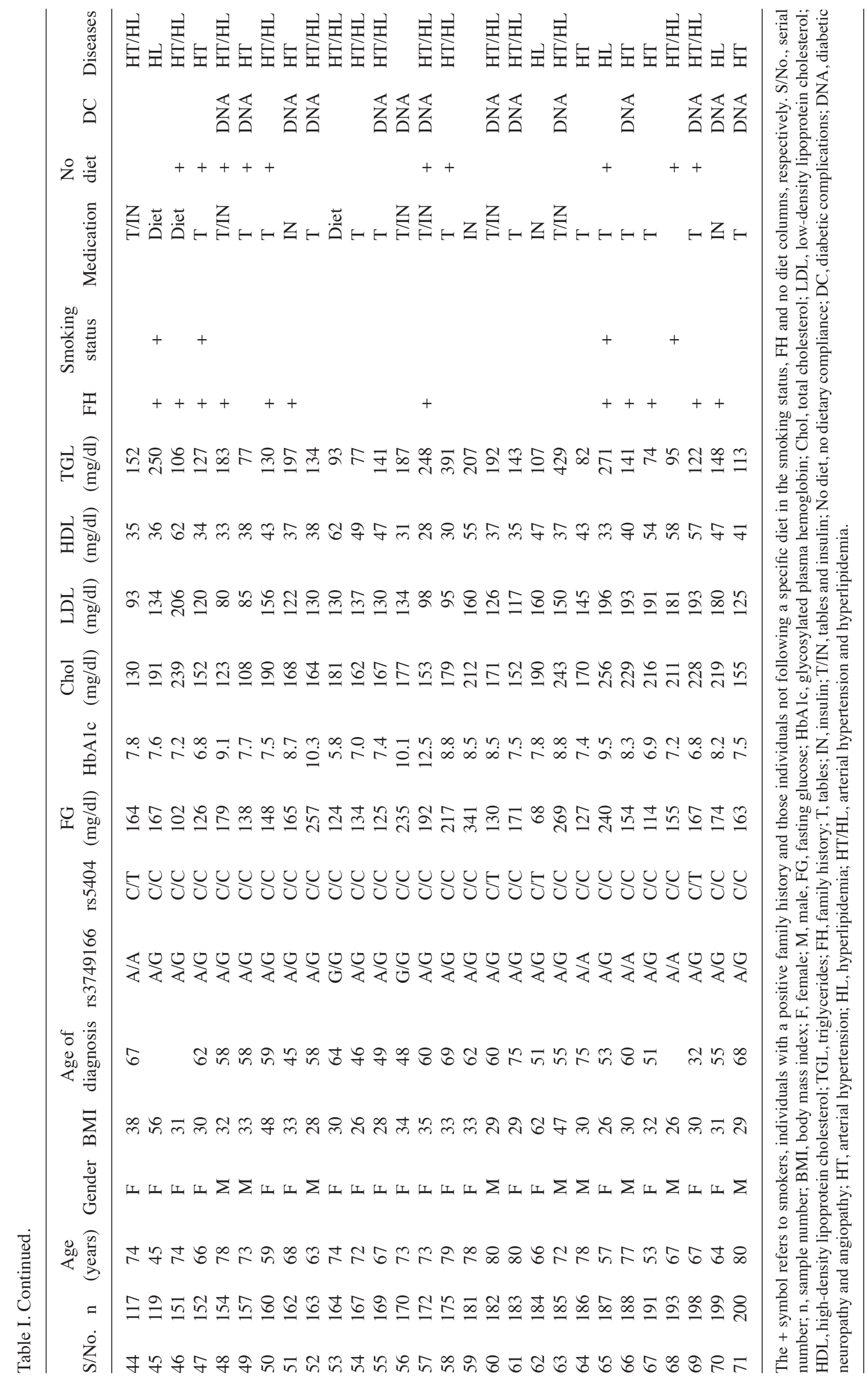


Table II. Statistical analysis of epidemiological parameters of individuals included in Table I.

\begin{tabular}{|c|c|c|c|c|}
\hline Parameter & $\begin{array}{c}\text { Non-diabetic } \\
\text { controls }^{\mathrm{a}}(\mathrm{n}=99)\end{array}$ & $\begin{array}{l}\text { T2D patients }{ }^{\mathrm{b}} \\
\quad(\mathrm{n}=71)\end{array}$ & $\chi^{2}$ & P-value \\
\hline Male & 24 & 21 & 0.605 & 0.4368 \\
\hline Female & 75 & 50 & & \\
\hline Age (years) & $60.62 \pm 10.11$ & $66.94 \pm 9.65$ & & $<0.0001$ \\
\hline Body mass index $\left(\mathrm{kg} / \mathrm{m}^{2}\right)$ & $28.96 \pm 5.34$ & $32.30 \pm 7.98$ & & 0.0013 \\
\hline Age of T2D diagnosis & & $56.37 \pm 10.95$ & & \\
\hline T2D duration (years) & & $11.65 \pm 8.33$ & & \\
\hline Fasting glucose (mg/dl) & $98.31 \pm 10.45$ & $170.32 \pm 6.63$ & & $<0.0001$ \\
\hline Glycosylated hemoglobin (\%) & $5.65 \pm 0.31$ & $8.13 \pm 1.21$ & & $<0.0001$ \\
\hline Total cholesterol (mg/dl) & $208.57 \pm 38.52$ & $188.96 \pm 40.46$ & & 0.0016 \\
\hline Low-density lipoprotein cholesterol (mg/dl) & $147.14 \pm 35.00$ & $169.78 \pm 36.81$ & & $<0.0001$ \\
\hline High-density lipoprotein cholesterol (mg/dl) & $50.93 \pm 13.06$ & $42.14 \pm 9.46$ & & $<0.0001$ \\
\hline Trigycerides $(\mathrm{mg} / \mathrm{dl})$ & $137.18 \pm 54.49$ & $179.45 \pm 134.09$ & & 0.0052 \\
\hline Family history (positive) & 6 & 25 & 23.565 & $<0.0001$ \\
\hline Smoking status & 20 & 10 & 1.065 & 0.3021 \\
\hline Not following dietary instructions & 13 & 20 & 5.977 & 0.0014 \\
\hline Diet & & 8 & & \\
\hline Medication (tablets) & & 35 & & \\
\hline Medication (insulin) & & 10 & & \\
\hline Medication (tablets and insulin) & & 13 & & \\
\hline No medication or dietary intervention & & 5 & & \\
\hline Diabetic complications (neuropathy, angiopathy) & & 30 & & \\
\hline Diabetic complications and disease duration ( $>4$ years) & & 24 & & 0.0014 \\
\hline Diabetic complications and disease duration ( $\leq 4$ years) & & 1 & & \\
\hline Accompanying diseases & 72 & 59 & 2.516 & 0.1127 \\
\hline Accompanying diseases (arterial hypertension) & 17 & 19 & 2.278 & 0.1313 \\
\hline Accompanying diseases (hyperlipidemia) & 24 & 12 & 1.335 & 0.2479 \\
\hline $\begin{array}{l}\text { Accompanying diseases (arterial hypertension } \\
\text { and hyperlipidemia) }\end{array}$ & 31 & 28 & 1.204 & 0.2725 \\
\hline
\end{tabular}

${ }^{a}$ Of the 48 PGP non-diabetic controls, 27 are male and 21 are female. The mean age of the 48 PGP non-diabetic controls with one or the two polymorphisms is 59.4 years $(\mathrm{SD}=10.5)$. ${ }^{\mathrm{b}} \mathrm{Of}$ the $23 \mathrm{PGP}$ T2D patients, 21 are male and 2 are female. The mean age of the 23 patients with one or the two polymorphisms is 56.7 years (SD=11.2). T2D, type 2 diabetes; PGP, Personal Genome Project; SD, standard deviation.

Austria) using the forward primer for rs3749166 and the reverse primer for rs5404). Nucleotide sequence analysis was performed using the Chromas software (version 2.6.2).

Personal Genome Project (PGP) data. To validate the results of the analysis, the allele frequencies of the rs5404 and rs3749166 polymorphisms were evaluated using public genome and exome data, available through the PGP repository (25). Cases matching the patient and non-diabetic control profiles of the present study were selected and 40 additional cases (35 non-diabetic controls and 5 T2D patients) were included, containing allele information of the rs3749166 polymorphism. In addition, 71 cases (48 non-diabetic controls and 23 T2D patients) with allele information of the rs5404 polymorphism were evaluated. Among these, 35 non-diabetic controls and 5 T2D patients contained genetic data for the two polymorphisms. Of the 71 PGP individuals, $32.4 \%$ were female and the mean age was 58 years (standard deviation, 10.85).
Statistical analysis. Graphpad online tool (https://www. graphpad.com) was used to perform statistical analyses. Student's t-test was used to compare groups of continuous variables, and the $\chi^{2}$ and Fisher's test were used to compare the proportions of genotypes or alleles. A two-tailed $\mathrm{P}<0.05$ was considered to indicate a statistically significant difference. The difference of the ESE scores between the major $\mathrm{S}_{\mathrm{A}}$ and minor $\mathrm{S}_{\mathrm{a}}$ alleles was calculated as the $\Delta \mathrm{Score}=\mid \mathrm{S}_{\mathrm{A}}-\mathrm{S}_{\mathrm{a}} \mathrm{l}$.

\section{Results}

Clinical data and statistical analysis of epidemiological parameters. Statistical analysis of the data from T2D patients and non-diabetic control subjects (Table II) demonstrated that among the T2D patients, fasting glucose levels and HbA1c were significantly higher $(\mathrm{P}<0.0001)$; however, there was no correlation with accompanying diseases, such as arterial hypertension or hyperlipidemia. By contrast, LDL and triglyceride 
Table III. Statistical evaluation of (A) rs3749166 and (B) rs5404 SNP frequencies and genotypes among total and Greek T2D patients and non-diabetic controls. (C) Association of observed rs3749166 and rs5404 genotype combinations with disease among total, and Greek T2D patients and non-diabetic control subjects.

A, rs3749166 genotypes

\begin{tabular}{|c|c|c|c|c|c|}
\hline Subject & $\mathrm{AA}, \mathrm{n}(\%)$ & $\mathrm{AG}, \mathrm{n}(\%)$ & GG, n (\%) & $\chi^{2}$ & P-value \\
\hline Non-diabetic control (total) & $40(29.85)$ & $71(52.99)$ & $23(17.16)$ & 11.09 & 0.0039 \\
\hline T2D patients (total) & $15(19.74)$ & $57(75.00)$ & $4(5.26)$ & & \\
\hline Non-diabetic control (Greek) & $29(29.29)$ & $54(54.55)$ & $16(16.16)$ & 7.28 & 0.0262 \\
\hline T2D patients (Greek) & $15(21.13)$ & $52(73.24)$ & $4(5.63)$ & & \\
\hline
\end{tabular}

B, rs5404 genotypes

\begin{tabular}{|c|c|c|c|c|c|}
\hline Subject & $\mathrm{TT}, \mathrm{n}(\%)$ & $\mathrm{CT}, \mathrm{n}(\%)$ & $\mathrm{CC}, \mathrm{n}(\%)$ & $\chi^{2}$ & P-value \\
\hline Non-diabetic control (total) & $0(0)$ & $51(34.69)$ & $96(65.31)$ & 4.967 & 0.0258 \\
\hline T2D patients (total) & $0(0)$ & $20(21.28)$ & $74(78.72)$ & & \\
\hline Non-diabetic control (Greek) & $0(0)$ & $39(39.39)$ & $60(60.61)$ & 5.369 & 0.0205 \\
\hline T2D patients (Greek) & $0(0)$ & $16(22.54)$ & $55(77.46)$ & & \\
\hline
\end{tabular}

C, rs3749166 and rs5404 combined genotypes

\begin{tabular}{|c|c|c|c|c|c|c|}
\hline Subject & $\begin{array}{c}\mathrm{GG} / \mathrm{CC}, \\
\mathrm{n}(\%)\end{array}$ & $\begin{array}{c}\mathrm{GG} / \mathrm{CT}, \\
\text { n }(\%)\end{array}$ & $\begin{array}{c}\mathrm{AG} / \mathrm{CC} \\
\mathrm{n}(\%)\end{array}$ & $\begin{array}{c}\mathrm{AG} / \mathrm{CT}, \\
\mathrm{n}(\%)\end{array}$ & $\begin{array}{c}\mathrm{AA} / \mathrm{CC} \\
\mathrm{n}(\%)\end{array}$ & $\begin{array}{c}\mathrm{AA} / \mathrm{CT}, \\
\mathrm{n}(\%)\end{array}$ \\
\hline Non-diabetic control (total) & $18(13.43)$ & $5(3.73)$ & $44(32.84)$ & $27(20.15)$ & $23(17.16)$ & $17(12.69)$ \\
\hline T2D patients (total) & $3(3.95)$ & $1(1.32)$ & $43(56.58)$ & $14(18.42)$ & $12(15.79)$ & $3(3.95)$ \\
\hline P-value & 0.031 & 0.4211 & $0.0008^{\mathrm{a}}$ & 0.7614 & 0.7973 & 0.0491 \\
\hline Non-diabetic control (Greek) & $14(14.14)$ & $2(2.02)$ & $29(29.29)$ & $25(25.25)$ & $17(17.17)$ & $12(12.12)$ \\
\hline T2D patients (Greek) & $3(4.22)$ & $1(1.40)$ & $40(56.33)$ & $12(16.90)$ & $12(16.90)$ & $3(4.22)$ \\
\hline P-value & 0.039 & 1.000 & $0.004^{\mathrm{b}}$ & 0.169 & 0.963 & 0.100 \\
\hline
\end{tabular}

P-values were evaluated with respect to the disease association of each genotype combination relative to the remaining genotype combinations. ${ }^{\mathrm{a}} \mathrm{OR}=2.67$; ${ }^{\mathrm{b}} \mathrm{OR}=3.11$. T2D, type 2 diabetes; OR, odds ratio.

levels were significantly higher among T2D patients $(\mathrm{P}<0.0001$; $\mathrm{P}=0.0052)$ and HDL levels were significantly lower $(\mathrm{P}<0.0001)$. The observed age difference among T2D patients and nondiabetic control subjects was significant $(\mathrm{P}<0.0001)$, potentially because the majority of individuals with T2D are diagnosed at an older age (data not shown). All other parameters, such as smoking status, did not differ among T2D patients and non-diabetic control subjects in the present study.

Genotype frequencies for rs3749166 and rs5404 SNPs in T2D patients and non-diabetic control subjects. The rs3749166 polymorphism was detected by PCR amplification (data not shown) and sequencing of a 153-bp PCR fragment, which included the SNP (Fig. 1A). Similarly, a 344-bp fragment, including the rs5404 SNP was amplified by PCR (data not shown) and analyzed by sequencing (Fig. 1B).

The rs3749166 and rs5404 frequencies for the Greek T2D patients and non-diabetic controls are summarized in Table III. Statistical analysis of these data revealed that only the heterozygous rs3749166 genotype (AG, partially epigenetic) was associated with T2D, while the epigenetic genotype $(\mathrm{GG})$ appeared to be protective for the disease $(\mathrm{P}=0.0262$; Table IIIA). A more significant positive correlation was obtained when the PGP data were incorporated into the study ( $\mathrm{P}=0.0039$; Table IIIA).

Analysis of the rs5404 polymorphism from the two sets of data revealed that the homozygous TT genotype was not observed, although the CT frequency was significant $(21.28 \%$ in T2D patients and $34.69 \%$ in non-diabetic control subjects) and that the $\mathrm{T}$ genotype may be protective for the disease (Table IIIB; $\mathrm{P}=0.0205$ and $\mathrm{P}=0.0258$ ).

Finally, the association of these splicing-affecting genotype combinations with TD2 was analyzed. The results are presented in Table IIIC and reveal that only the AG/CC genotype is strongly associated with $\mathrm{T} 2 \mathrm{D}$ in all cases examined [Greek: $\mathrm{P}=0.004$ and odds ratio (OR), 3.11; $\mathrm{PGP}: \mathrm{P}=0.0008$ and OR, 2.67]. Furthermore, the GG/CC and AG/CT genotypes may be protective for the disease. In addition, the $\mathrm{T}$ allele was infrequent among individuals who were homozygous for rs3749166 (GG/CT epigenetic genotype). 


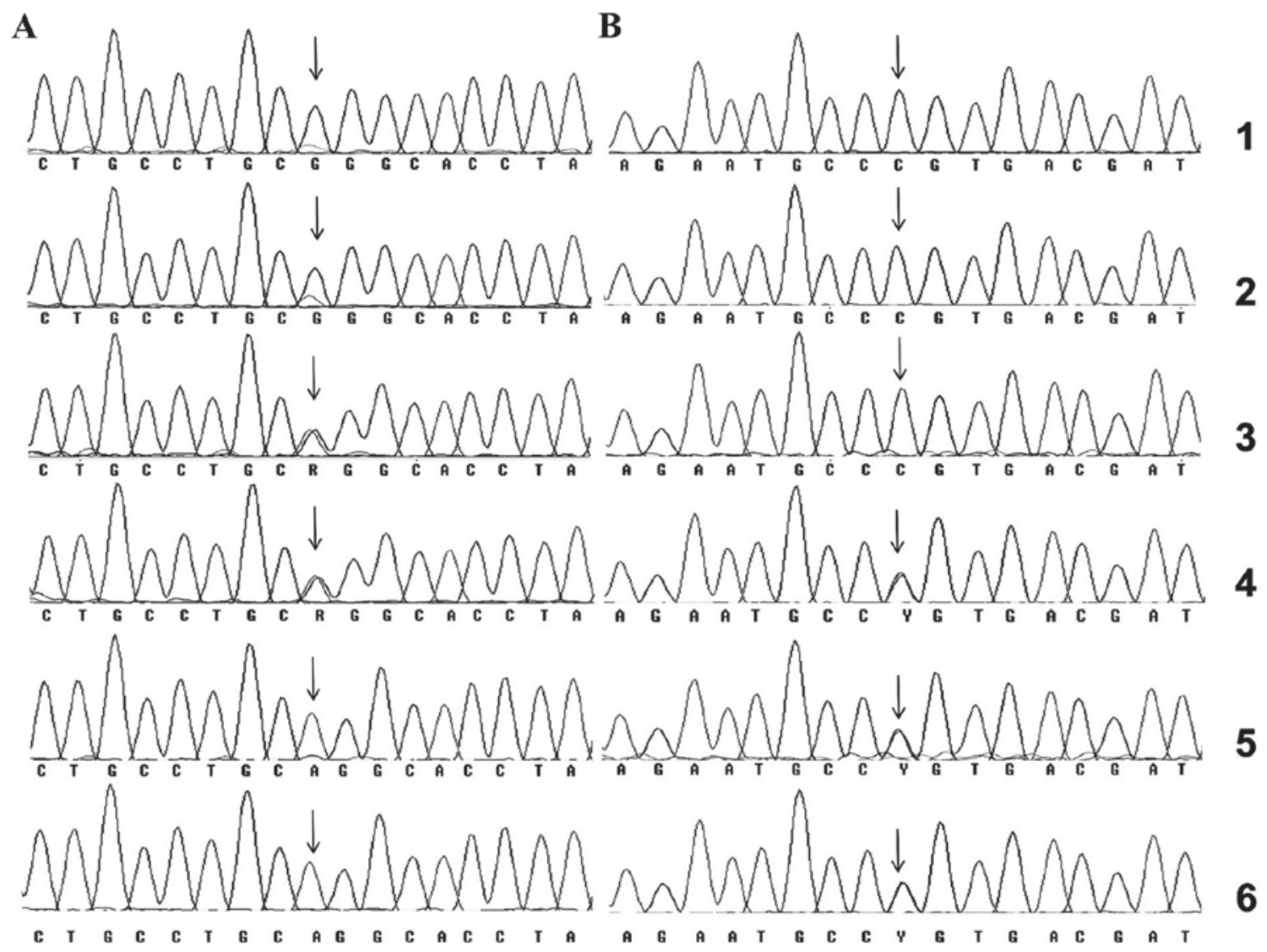

Figure 1. (A) Nucleotide sequence of the amplified fragments containing the rs3749166 (A>G) calpain 10 polymorphism for samples $34,40,187,200,46$ and 117 (rows 1-6, respectively). (B) Nucleotide sequences of the amplified fragments of rs5404 (C>T) solute carrier family 2 member 2 for the samples 3, 68, 74, 19, 47 and 77 (rows 1-6, respectively). rs3749166 and rs5404 polymorphic sites are indicated using arrows.

Table IV. AG/CC genotype combination among all individuals and T2D patients, relative to the HbA1c levels ( $\geq 8.5 \%)$. T2D patients are shown in parenthesis.

\begin{tabular}{llccccc}
\hline & AG/CC & AA/CC & GG/CC & AG/CT & AA/CT & GG/CT \\
\hline HbAlc $\geq 8.5 \%$ & $19(19)$ & $3(3)$ & $1(1)$ & $3(3)$ & 0 & 0 \\
HbA1c $<8.5 \%$ & $50(21)$ & $26(9)$ & $16(2)$ & $34(9)$ & $15(3)$ & $3(1)$ \\
Total & $69(40)^{\mathrm{a}}$ & $29(12)$ & $17(3)$ & $37(12)$ & $15(3)$ & $3(1)$ \\
\hline
\end{tabular}

${ }^{\mathrm{a}} \mathrm{P}=0.0002, \mathrm{OR}=5.10(\mathrm{P}=0.0306, \mathrm{OR}=1.30)$. T2D, type 2 diabetes; HbA1c, glycosylated plasma hemoglobin; OR, odds ratio.

Association of the rs3749166/rs5404 genotype combinations with glucose metabolism. Another common characteristic among carriers of the AG/CC genotype (disease-associated) is the presence of high HbAlc levels $(\geq 8.5 \%)$ (Table IV; $\mathrm{P}=0.0002$, OR, 5.10) although neither of the polymorphisms was found to be independently associated with the T2D criteria (elevated fasting glucose levels and HbA1c).

\section{Discussion}

Elucidating the impact of epigenetic synonymous SNPs, particularly those involved in the regulation of alternatively spliced exons, is critical for understanding the pathogenesis of complex diseases. The polymorphisms included in the present study were selected on the basis of their epigenetic character and because they are the only synonymous SNPs strongly modifying the splicing-associated exonic enhancers associated with glucose transport (11). CAPN10 and GLUT2 participate in complementary transporter systems, which might be expected to act in a concerted manner. CAPN10 is a T2D-associated protease, which facilitates insulin-stimulated GLUT4 translocation via its activity on the distal secretory pathway (16). Although the association of rs3749166 with T2D has been the subject of various reviews and meta-analytic studies, it is still questioned if it may influence the development of T2D independently or in combination with other CAPN10 gene polymorphisms $(17,18)$. Furthermore, the second SNP investigated in the present study, rs5404 in SLC2A2, has been evaluated in association with T2D (19-22); however, the obtained results were contradictory. In certain studies $(20,21)$ a 
Table V. Evaluation of the ESE modifications introduced by the rs3749166 (A>G) and rs5404 (C>T) SNPs using ESE finder [Cartegni et al (4)].

\begin{tabular}{|c|c|c|c|c|c|c|}
\hline Gene & SNP & Exon type & $\begin{array}{c}\text { Splice site/SR } \\
\text { protein binding }\end{array}$ & $\begin{array}{l}\text { Major allele ESE } \\
\text { finder score }\end{array}$ & $\begin{array}{l}\text { Minor allele ESE } \\
\text { finder score }\end{array}$ & $\Delta$ Score $^{\mathrm{a}}$ \\
\hline CAPN10 & rs3749166 & Alternative & $\begin{array}{l}\text { Exon } 11 \text { splice site } \\
\text { (3SS_U2_human) }\end{array}$ & 10.350 & -3.220 & 13.570 \\
\hline SLC2A2 & rs5404 & Constitutive & $\begin{array}{l}\text { SRp40 } \\
\text { SF2/ASF (IgM-BRCA1) } \\
\text { SF2/ASF }\end{array}$ & $\begin{array}{l}3.793 \\
2.337 \\
3.162\end{array}$ & $\begin{array}{l}6.324 \\
3.769 \\
3.778\end{array}$ & $\begin{array}{l}2.531 \\
1.402 \\
0.616\end{array}$ \\
\hline
\end{tabular}

${ }^{\mathrm{a}} \Delta$ Score: Difference of the ESE scores between the major and minor allele. Lower splicing score limit according to ESE finder: 3SS_U2 human=6.632; SRp40=2.670; SF2/ASF(IgM-BRCA1)=1.867; SF2/ASF=1.956. ESE, exonic splicing enhancers; SNP, single nucleotide polymorphism; CAPN10, calpain 10; SLC2A2, solute carrier family 2, member 2.

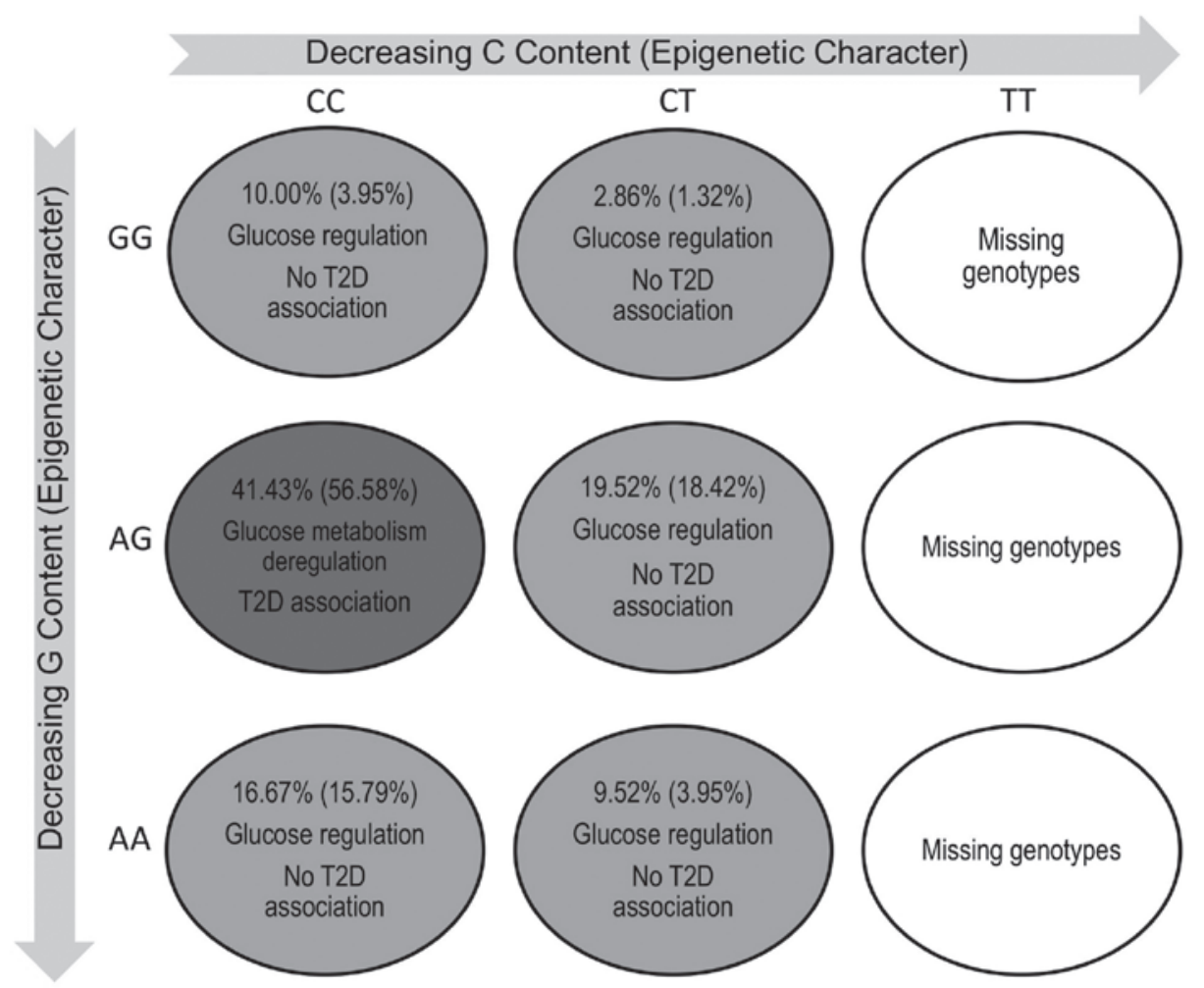

Figure 2. Frequencies of combined observed and expected genotypes resulting from rs3749166 and rs5404 single nucleotide polymorphism in all analyzed cases. The T2D patient frequencies are presented in parenthesis. The genotypes are presented in order of decreasing epigenetic character. Missing combined genotypes are also included. T2D, type 2 diabetes.

significant risk was observed among homozygotes, which was similar to the present results, while in another study (19) the minor allele was found to be associated with increased disease risk and with reduced postprandial glucose levels. To the best of our knowledge, the present study is the first to provide a comprehensive concurrent analysis of two SNPs (rs3749166 and rs5404) under investigation, which appear to be critical for the splicing of genes involved in complementary glucose transport systems.

Computational analysis has shown that the two polymorphisms interfere with splicing regulation (11). However, according to the data reported by Karambataki et al (11) and summarized in Table V (4), these SNPs modify the binding potential of splicing factors in different ways. rs3749166 (A allele) in CAPN10 strongly modifies the binding site of 3SS_U2 splicing enhancer of alternatively expressed exon 11 and, thus, may lead to the production of more than one splicing product in AG heterozygotes. By contrast, in its heterozygotic state, rs5404 (T allele) in SLC2A2 modifies the response of the ESE elements in this sequence to serine/arginine-rich (SR) proteins, particularly SRp40 and SF2/ASF (IgM-BRCA1) (26). As the two SNPs modify CpG sequences, they also perturb epigenetic regulation for the homozygotic genotypes AA and TT (but not GG and CC, or AG and CT genotypes); however, they do not introduce functionally significant single amino acid modifications (coding synonymous). 
The present results, summarized in Fig. 2, indicate that the most protective genotype for T2D is the fully epigenetic genotype. In accordance with the above-mentioned analysis, the heterozygous rs3749166 and rs5404 genotypes are also differently associated with T2D. Carriers of the AG/CC genotype (heterozygous for rs3749166) are significantly more frequent among the T2D patients and exhibit particularly high levels of HbAlc, probably indicating resistance to pharmaceutical intervention for T2D. Similar findings regarding the negative effect associated with the synthesis of two different isoforms have been recently reported in association with heterozygous SNPs causing alternative splicing. For example, Tian et al (27) reported a number of disorders associated with alternative exon expression and splicing. In addition, Kurzawski et al (14) reported on the effect of epigenetic SNP rs5030952 in CAPN10, which exhibits a heterozygotic association with post-transplant diabetes mellitus.

By contrast, the presence of the apparently protective rs5404 SNP (CT genotype in SLC2A2) is potentially associated with the modified response of $\mathrm{CT}$ heterozygotes to different stimuli (based on the data from ESE score analysis at least one novel ESE is formed, which significantly responds to SRp40 proteins). This appears to be particularly significant for carriers of the rs3749166 CAPN10 AG genotype (the combined AG/CT genotype is not T2D-associated, and its carriers do not exhibit particularly high levels of HbA1c. The advantage of epigenetic regulation provided by the $\mathrm{C}$ allele and, thus, the ESE response may be lost among TT homozygotes. This could be a possible explanation for the absence of TT homozygotes regardless of the relatively high total frequency of the $\mathrm{T}$ allele (21.28\%, non-Mendelian genetics).

These findings provide the hypothesis that mutations modifying the response to splicing regulatory mechanisms (epigenetic and ESE) may be associated with strong negative functional changes, and exhibit complex, nonlinear disease associations (28). Provided that functionally significant epigenetic SNPs are frequent (11), this type of genetic variation is expected to have a strong impact on disease and evolution.

A major obstacle in investigating complex pathological conditions, such as metabolic syndrome, is the limited understanding of the regulatory factors involved in the expression of interacting components. Recent evidence indicates the key role of alternative RNA expression in developmental changes (29), and the production of coding and non-coding RNA sequences. Another factor is the complex epigenetic modifications, which may also lead to the expression of different RNA isoforms. The current results indicate likely synergies between synonymous splicing-regulatory epigenetic SNPs, which modify the splicing potential of two different glucose transport-associated genes, and reveal that bioinformatic analysis and careful investigation of the SNPs under investigation may become a powerful tool for identifying potentially significant genetic modifications with respect to splicing.

In conclusion, the results presented above indicate for the first time, to the best of our knowledge, the correlation and disease association of two synonymous epigenetic SNPs, which participate in the regulation of the glucose transport system and introduce exclusively splicing-associated modifications. Taken together, these results reveal that T2D is subject to deregulation by complex splicing mechanisms, which may exhibit heterozygous disease association or protection, depending on the splicing-affecting genetic variation. A detailed bioinformatic analysis of the changes introduced by SNPs would facilitate the understanding of the impact of functional changes introduced by genetic variation.

\section{Acknowledgements}

The present study was co-financed by the European Union (the European Social Fund) and Greek national funds through the Operational Program, 'Education and Lifelong Learning' of the NSRF - Research Funding Program: Heracleitus II. Investing in knowledge society through the European Social Fund (project no. 87113).

\section{References}

1. Romero PR, Zaidi S, Fang YY, Uversky VN, Radivojac P, OldfieldCJ,Cortese MS,SickmeierM,LeGallT,ObradovicZ,etal: Alternative splicing in concert with protein intrinsic disorder enables increased functional diversity in multicellular organisms. Proc Natl Acad Sci USA 103: 8390-8395, 2006.

2. Stamm S, Ben-Ari S, Rafalska I, Tang Y, Zhang Z, Toiber D, Thanaraj TA and Soreq H: Function of alternative splicing. Gene 344: 1-20, 2005

3. Soukarieh O,GaildratP,Hamieh M, Drouet A,Baert-Desurmont S, Frébourg T, Tosi M and Martins A: Exonic splicing mutations are more prevalent than currently estimated and can be predicted by using in silico tools. PLoS Genet 12: e1005756, 2016.

4. Cartegni L, Wang J, Zhu Z, Zhang MQ and Krainer AR: ESEfinder: a web resource to identify exonic splicing enhancers. Nucleic Acids Res 31: 3568-3571, 2003.

5. Anastasiadou C, Malousi A, Maglaveras N and Kouidou S: Human epigenome data reveal increased $\mathrm{CpG}$ methylation in alternatively spliced sites and putative exonic splicing enhancers. DNA Cell Biol 30: 267-275, 2011.

6. Ong CT and Corces VG: CTCF: an architectural protein bridging genome topology and function. Nat Rev Genet 15: 234-246, 2014.

7. Malousi A and Kouidou S: DNA hypermethylation of alternatively spliced and repeat sequences in humans. Mol Genet Genomics 287: 631-642, 2012.

8. Shoemaker R, Deng J, Wang W and Zhang K: Allele-specific methylation is prevalent and is contributed by CpG-SNPs in the human genome. Genome Res 20: 883-889, 2010.

9. Scalet D, Balestra D, Rohban S, Bovolenta M, Perrone D, Bernardi F, Campaner $\mathrm{S}$ and Pinotti $\mathrm{M}$ : Exploring splicing-switching molecules for seckel syndrome therapy. Biochim Biophys Acta 1863: 15-20, 2016.

10. Karambataki M, Malousi A, Maglaveras N and Kouidou S: Synonymous polymorphisms at splicing regulatory sites are associated with $\mathrm{CpGs}$ in neurodegenerative disease-related genes. Neuromolecular Med 12: 260-269, 2010.

11. Karambataki M, Malousi A and Kouidou S: Risk-associated coding synonymous SNPs in type 2 diabetes and neurodegenerative diseases: Genetic silence and the underrated association with splicing regulation and epigenetics. Mutat Res 770: 85-93, 2014.

12. Harlid S, Ivarsson MI, Butt S, Hussain S, Grzybowska E, Eyfjörd JE, Lenner P, Försti A, Hemminki K, Manjer J, et al: A candidate CpG SNP approach identifies a breast cancer associated ESR1-SNP. Int J Cancer 129: 1689-1698, 2011.

13. Imamura M and Maeda S: Genetics of type 2 diabetes: the GWAS era and future perspectives (Review). Endocr J 58: 723-739, 2011.

14. Kurzawski M,DziewanowskiK,KedzierskaK,Gornik W,Banas A and Drozdzik M: Association of calpain-10 gene polymorphism and posttransplant diabetes mellitus in kidney transplant patients medicated with tacrolimus. Pharmacogenomics J 10: 120-125, 2010.

15. Shchetynsky K, Protsyuk D, Ronninger M, Diaz-Gallo LM, Klareskog L and Padyukov L: Gene-gene interaction and RNA splicing profiles of MAP2K4 gene in rheumatoid arthritis. Clin Immunol 158: 19-28, 2015. 
16. Brown AE, Yeaman SJ and Walker M: Targeted suppression of calpain-10 expression impairs insulin-stimulated glucose uptake in cultured primary human skeletal muscle cells. Mol Genet Metab 91: 318-324, 2007.

17. Alsaraj F, O'Gorman D, McAteer S, McDermott J, Hawi Z and Sreenan S: Haplotype association of calpain 10 gene variants with type 2 diabetes mellitus in an Irish sample. Ir J Med Sci 179: 269-272, 2010

18. Song Y, You NC, Hsu YH, Sul J, Wang L, Tinker L, Eaton CB and Liu S: Common genetic variation in calpain-10 gene (CAPN10) and diabetes risk in a multi-ethnic cohort of American postmenopausal women. Hum Mol Genet 16: 2960-2971, 2007.

19. Barroso I, Luan J, Middelberg RP, Harding AH, Franks PW, Jakes RW, Clayton D, Schafer AJ, O'Rahilly S and Wareham NJ: Candidate gene association study in type 2 diabetes indicates a role for genes involved in beta-cell function as well as insulin action. PLoS Biol 1: E20, 2003.

20. Laukkanen O, Lindström J, Eriksson J, Valle TT, Hämäläinen H, Ilanne-Parikka P, Keinänen-Kiukaanniemi S, Tuomilehto J, Uusitupa M and Laakso M; Finnish Diabetes Prevention Study: Polymorphisms in the SLC2A2 (GLUT2) gene are associated with the conversion from impaired glucose tolerance to type 2 diabetes: The Finnish Diabetes Prevention Study. Diabetes 54 2256-2260, 2005.

21. Kilpeläinen TO, Lakka TA, Laaksonen DE, Mager U, Salopuro T, Kubaszek A, Todorova B, Laukkanen O, Lindström J, Eriksson JG, et al; Finnish Diabetes Prevention Study Group: Interaction of single nucleotide polymorphisms in ADRB2, ADRB3, TNF, IL6, IGF1R, LIPC, LEPR, and GHRL with physical activity on the risk of type 2 diabetes mellitus and changes in characteristics of the metabolic syndrome: The Finnish Diabetes Prevention Study. Metabolism 57: 428-436, 2008
22. Willer CJ, Bonnycastle LL, Conneely KN, Duren WL, Jackson AU, Scott LJ, Narisu N, Chines PS, Skol A, Stringham HM, et al: Screening of 134 single nucleotide polymorphisms (SNPs) previously associated with type 2 diabetes replicates association with 12 SNPs in nine genes. Diabetes 56: 256-264, 2007.

23. American Diabetes Association: Standards of medical care in diabetes - 2013. Diabetes Care 36 (Suppl 1): S11-S66, 2013.

24. Ye J, Coulouris G, Zaretskaya I, Cutcutache I, Rozen S and Madden TL: Primer-BLAST: A tool to design target-specific primers for polymerase chain reaction. BMC Bioinformatics 13: 134, 2012.

25. Church GM: The personal genome project. Mol Syst Biol 1: 2005.0030, 2005.

26. Chen HH, Wang YC and Fann MJ: Identification and characterization of the CDK12/cyclin L1 complex involved in alternative splicing regulation. Mol Cell Biol 26: 2736-2745, 2006.

27. Tian C, Yan R, Wen S, Li X, Li T, Cai Z, Li X, Du H and Chen H: A splice mutation and mRNA decay of EXT2 provoke hereditary multiple exostoses. PLoS One 9: e94848, 2014.

28. Strohman RC: Linear genetics, non-linear epigenetics: complementary approaches to understanding complex diseases. Integr Physiol Behav Sci 30: 273-282, 1995.

29. Lau E: Non-coding RNA: Zooming in on lncRNA functions. Nat Rev Genet 15: 574-575, 2014. 